\title{
Comparative immune responses of pups following modified live virus vaccinations against canine parvovirus
}

\author{
Jayalakshmi Vasu ${ }^{1}$, Mouttou Vivek Srinivas ${ }^{1}$, Prabhakar Xavier Antony ${ }^{1}$, Jacob Thanislass ${ }^{2}$, Vijayalakshmi Padmanaban ${ }^{3}$
} and Hirak Kumar Mukhopadhyay ${ }^{1}$

1. Department of Veterinary Microbiology, Rajiv Gandhi Institute of Veterinary Education and Research, Puducherry, India; 2. Department of Veterinary Biochemistry, Rajiv Gandhi Institute of Veterinary Education and Research, Puducherry, India; 3. Department of Veterinary Medicine, Rajiv Gandhi Institute of Veterinary Education and Research, Puducherry, India.

Corresponding author: Hirak Kumar Mukhopadhyay, e-mail: mhirak@rediffmail.com

Co-authors: JV: jaya591@gmail.com, MVS: vivekvet24@gmail.com, PXA: pxantony@gmail.com, JT: jthanislass@gmail.com, VP: vijinethira@gmail.com

Received: 14-05-2019, Accepted: 31-07-2019, Published online: 17-09-2019

doi: 10.14202/vetworld.2019.1422-1427 How to citethis article: Vasu J, Srinivas MV, Antony PX, Thanislass J, Padmanaban V, Mukhopadhyay HK (2019) Compaative immune responses of pups following modified live virus vaccinations against canine parvovirus, Veterinary World, 12(9): 1422-1427.

\begin{abstract}
Background and Aim: Canine parvovirus (CPV) is the most important viral cause of enteritis and mortality in pups. Evaluation and monitoring of pre- and post-vaccine immune responses may help to determine the efficacy of the current vaccination schedule being followed in pups in India. This study aimed to evaluate and monitor the pre- and post-vaccine immune responses of CPV vaccinated pups using hemagglutination inhibition (HI) assay. The neutralizing antibody titer levels were also detected using serum neutralization test (SNT).
\end{abstract}

Materials and Methods: The pups were categorized into two groups, the double booster and the single booster groups. In this study, serum samples were subjected to HI and SNT for measuring the CPV antibody titer at frequent intervals for up to 6 months from 27 healthy pups following primary and booster CPV vaccinations.

Results: The antibody titers in double booster pups reached their peaks at the $21^{\text {st }}$ day after the second booster vaccination with a geometric mean (GM) of 3.57. The antibody titers in single booster pups reached their peaks at the $21^{\text {st }}$ day after the first booster vaccination with a lower GM of 3.18.

Conclusion: The double booster pups maintained a higher immune response throughout the period of the study compared to single booster pups though the difference in titers was not statistically significant. SNT results indicated that the raised antibody titer was also able to yield virus-neutralizing antibodies. No interfering maternally derived antibodies were found in the pups at the age of primary vaccination $\left(45^{\text {th }}\right.$ day) in our study. Therefore, the second booster vaccination may be useful in maintaining the protective titer for a prolonged period.

Keywords: canine parvovirus, hemagglutination inhibition test, serum neutralization test, vaccine immune responses.

\section{Introduction}

Canine parvovirus (CPV) is an important pathogenic virus and is the causative agent of acute hemorrhagic enteritis and myocarditis in dogs. It is highly contagious and often a fatal disease. CPV-2 was first recognized in 1977 and since then, it has been well established as an enteric pathogen of dogs throughout the world with high morbidity (100\%) and frequent mortality up to $10 \%$ [1]. After the emergence of CPV in the late 1970s [1,2], due to rapid evolution, new antigenic types were evolved as CPV-2a, CPV-2b (CPV2a with Asn426Asp), and CPV-2c (CPV2a with Asn426Glu) and have completely replaced the original CPV-2 [3]. At present, the original CPV-2 is not found in dog population but present only in vaccine

Copyright: Vasu, et al. Open Access. This article is distributed under the terms of the Creative Commons Attribution 4.0 International License (http://creativecommons.org/licenses/by/4.0/), which permits unrestricted use, distribution, and reproduction in any medium, provided you give appropriate credit to the original author(s) and the source, provide a link to the Creative Commons license, and indicate if changes were made. The Creative Commons Public Domain Dedication waiver (http://creativecommons.org/ publicdomain/zero/1.0/) applies to the data made available in this article, unless otherwise stated. formulations $[3,4]$. Commonly, CPV causes disease in unvaccinated 1-6-month-old pups. Vaccinated pups are usually protected from the disease and from infection, unless immunization fails due to the presence of high titers of maternally derived antibodies (MDAs) [5,6]. The pups with MDA titers $\geq 1: 20$ may fail to be immunized successfully and remain susceptible to infection. Maternally derived hemagglutination inhibition (HI) antibody titers $\geq 1: 80$ are believed to protect pups from infection, while titers between 1:20 and 1:80 are too low to provide protection, but high enough to prevent active immunization [7]. Earlier, one CPV-2b based vaccine was available in India, but now only CPV-2 based vaccines are widely used for immunoprophylaxis of pups. Recent studies showed that the CPV strains isolated from the field are clustering away from the vaccine strains [8]. Moreover, an increasing proportion of CPV vaccinated dogs was found susceptible to infection in India raising serious concern about the efficacy of the vaccine strains and vaccination process [9].

Most puppies in many countries are first vaccinated with a multivalent vaccine against $\mathrm{CPV}$, canine 
distemper virus, canine adenovirus type 2 , canine parainfluenza virus, and leptospirosis when they are between 6 and 8 weeks of age, with booster vaccinations being given every 3-4 weeks until the age of 16 weeks and possibly 24 weeks in high-risk breeds. All the dogs should receive a booster 1 year after completion of the initial series followed by booster every 3 years [10]. It should be a common practice to avoid direct or indirect contact with potential sources of infection such as other dogs until 1-2 weeks after completion of this course of vaccination.

Additional inoculation of adult dogs with these vaccines is performed annually or as required by the veterinarians' experience, the rearing environment and the owner's wish in the hope of a booster effect in the animal's antibody titer [11]. In addition, some owners and veterinarians erroneously hold the view that infectious diseases such as parvovirus infection can be controlled by frequent vaccination alone [12] and perceive all vaccination programs to be protective for puppies under all circumstances.

Concerns regarding the influence of MDA have also prompted general opposition by practitioners to vaccination at early ages. Both high-titer CPV vaccines and intranasal vaccine administration proved to be good strategies to overcome the problem of MDA interference $[7,13]$. However, evaluation of the MDA levels in pups would be a more precise approach, establishing the appropriate time for vaccination on the basis of the actual immune status of the animals, rather than relying on a standardized schedule [14]. Evaluation and monitoring of post-vaccine immune responses may also help to determine the efficacy of the current vaccination schedule being followed in pups in India.

This study aimed to evaluate and monitor the pre- and post-vaccine immune responses of CPV vaccinated pups using $\mathrm{HI}$ assay. The neutralizing antibody titer levels were also detected using serum neutralization test (SNT).

\section{Materials and Methods}

\section{Ethical approval}

Ethical approval was not necessary for this study. However, samples were collected as per standard collection procedure without harming or giving stress to the animals.

\section{Collection of serum samples}

The pups were categorized into two groups, the single booster (11) and double booster (16) group (Table-1). In the single booster group, the pups were given the primary vaccine (42-45-day-old pups) followed by a single booster dose (after 21 days), whereas in the double booster pups in addition to the above vaccination, another booster dose was given (21 days after the first booster) (Table-1). A commercial multivalent vaccine (containing CPV-2 strain) was used for vaccinating the pups.

Blood samples were collected from all the vaccinated pups on the day of primary CPV vaccination
Table-1: Details of the puppies under the study.

\begin{tabular}{llll}
\hline Dog & Breed & Sex & $\begin{array}{l}\text { Dam's vaccination } \\
\text { status }\end{array}$ \\
\hline Single booster group & & \\
\hline Dog 1 & Labrador retriever & Male & Unknown \\
Dog 2 & Doberman & Female & Vaccinated regularly \\
Dog 3 & Doberman & Male & Vaccinated regularly \\
Dog 4 & Labrador retriever & Male & Vaccinated regularly \\
Dog 5 & Mongrel & Male & Unknown \\
Dog 6 & Doberman & Male & Unknown \\
Dog 7 & GSD & Female & Unknown \\
Dog 8 & Rajapalayam & Male & Unknown \\
Dog 9 & Chippipaarai & Male & Unknown \\
Dog 10 & Labrador retriever & Male & Unknown \\
Dog 11 & Mongrel & Female & Unknown \\
\hline Double booster group & & \\
\hline Dog 12 & Mongrel & Male & Unknown \\
Dog 13 & GSD & Male & Vaccinated regularly \\
Dog 14 & GSD & Female & Vaccinated regularly \\
Dog 15 & Labrador retriever & Female & Unknown \\
Dog 16 & Mongrel & Male & Unknown \\
Dog 17 & Mongrel & Female & Vaccinated regularly \\
Dog 18 & GSD & Male & Vaccinated regularly \\
Dog 19 & Dalmatian & Male & Vaccinated regularly \\
Dog 20 & Mongrel & Male & Unknown \\
Dog 21 & Rajapalayam & Male & Vaccinated regularly \\
Dog 22 & GSD & Male & Vaccinated regularly \\
Dog 23 & Mongrel & Female & Vaccinated regularly \\
Dog 24 & Mongrel & Male & Unknown \\
Dog 25 & Alsatian & Female & Unknown \\
Dog 26 & Alsatian & Female & Unknown \\
Dog 27 & Mongrel & Male & Unknown \\
\hline & & & \\
& & & \\
\hline
\end{tabular}

(considered as 0 day), followed by the $21^{\text {st }}$ day (first booster vaccination), $42^{\text {nd }}$ day (second booster vaccination), $63^{\text {rd }}$ day, $84^{\text {th }}$ day, $105^{\text {th }}$ day, $126^{\text {th }}$ day, $147^{\text {th }}$ day, and $168^{\text {th }}$ day post-primary vaccination. The collected blood samples were allowed to clot. Then, the tubes were centrifuged and the serum was collected and stored at $-20^{\circ} \mathrm{C}$ until further testing.

\section{HI test}

Two-fold serial dilutions of $25 \mu 1$ of serum samples were made in $25 \mu \mathrm{l}$ of $0.2 \mathrm{M}$ Sorenson's phosphate-buffered saline (PBS) $(\mathrm{pH} 7.0)$ followed by the addition of $4 \mathrm{HA}$ units of $\mathrm{CPV}-2$ vaccine strain. A commercial vaccine containing CPV-2 strain ( $>10^{7.0}$ $\mathrm{TCID}_{50} /$ dose) was used for preparing $4 \mathrm{HAU}$. The plates were incubated at $37^{\circ} \mathrm{C}$ for $45 \mathrm{~min}$ followed by $4^{\circ} \mathrm{C}$ for $30 \mathrm{~min}$. Then, $50 \mu \mathrm{l}$ of $0.65 \%$ of pig erythrocytes were added with gentle mixing and left at $4{ }^{\circ} \mathrm{C}$ for $1 \mathrm{~h}$. Cell controls in the form of $50 \mu \mathrm{l}$ of $0.2 \mathrm{M}$ Sorenson's PBS and $50 \mu 1$ of $0.65 \%$ of pig erythrocytes and virus controls with $25 \mu 1$ of $0.2 \mathrm{M}$ Sorenson's PBS, $25 \mu \mathrm{l}$ of $4 \mathrm{HA}$ units of virus, and $50 \mu 1$ of $0.65 \%$ of pig erythrocytes and serum controls with $25 \mu 1$ of $0.2 \mathrm{M}$ Sorenson's PBS, $25 \mu \mathrm{l}$ of serum samples, and $50 \mu 1$ of $0.65 \%$ of pig erythrocytes were also kept [15]. The HI titer was expressed as the reciprocal of the highest dilution of the serum that completely inhibited the HA activity. The $\log _{10}$ geometric mean (GM) was calculated by converting the HI titers into $\log _{10}$ values 
(X) and using the formula $\mathrm{GM}=\sqrt[n]{\mathrm{X}_{1} \times \mathrm{X}_{2} \times \mathrm{X}_{3} \times \ldots \mathrm{X}_{\mathrm{n}}}$, where $\mathrm{n}=$ total number of pups. The data analysis was performed using the MS Office Excel 2007 software.

SNT

Fifty microliter of chilled DMEM was added to the wells labeled as A1-A12. Using a sterile tip, $50 \mu \mathrm{l}$ of test serum was added to the first well (A1), thus making a serum dilution to $1: 2$ and mixed well by pipetting. Serial two-fold dilutions of test serum were made from A1 to A12. Wells were mixed after each transfer and continued dilution until the end. After the final dilution, $50 \mu 1$ was discarded. Using a sterile tip, an equal volume $(50 \mu \mathrm{l})$ containing $100 \mathrm{TCID}_{50}$ of the virus (CPV-2a strain maintained in the Department of VMC, RIVER, Puducherry) was added to each well of the serum dilution. The plates were incubated at $37^{\circ} \mathrm{C}$ for $1 \mathrm{~h}$. After incubation, $100 \mu \mathrm{l}$ of a suspension containing 20,000 CRFK cells were added to the above virus-serum mixture. The cells were distributed evenly by rocking the plate back and forth and from side to side. Then, the plates were incubated at $37^{\circ} \mathrm{C}$ for

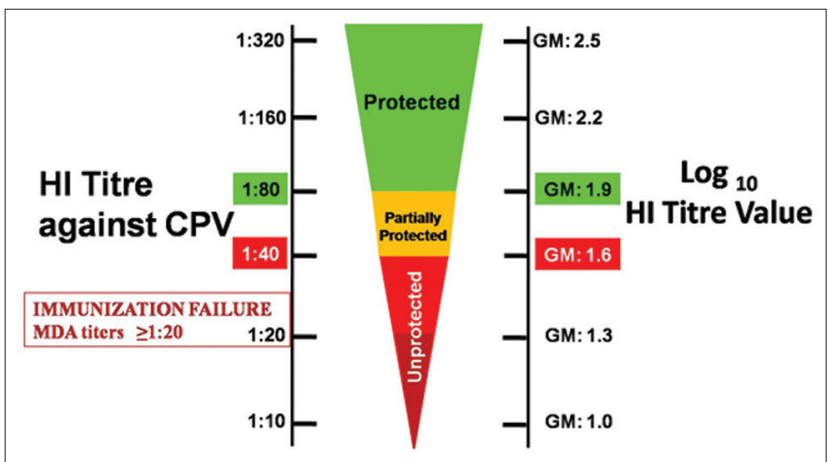

Figure-1: Hemagglutination inhibition (HI) titer (left side) and $\log 10 \mathrm{HI}$ titer scale (right side) to determine the protection, partial protection, and unprotected pups against canine parvovirus.
5 days in $3.5 \%$ humidified $\mathrm{CO}_{2}$ incubator. Following incubation, the plates were subjected to three freezethaw cycles. The undiluted cryolysate was centrifuged at $6000 \mathrm{rpm}$ at $4^{\circ} \mathrm{C}$ for $10 \mathrm{~min}$, and the supernatants were tested for CPV hemagglutination activity using freshly prepared $0.65 \%$ of pig erythrocytes [16].

Neutralizing antibody titers were calculated as the reciprocal of the highest serum dilution that completely neutralized the virus (absence of HA activity). The $\log _{10}$ GM was calculated by converting the SNT titers into $\log _{10}$ values. The statistical analysis was performed using the t-test. $\mathrm{p} \leq 0.05$ was considered statistically significant. The data analysis was performed using the MS Office Excel 2007 software (Microsoft, USA).

\section{Results and Discussion}

\section{HI test}

No interfering MDA was detected by HI test during primary vaccination ( 0 day), i.e., at the age of the $45^{\text {th }}$ day of the pups. However, at the same time, the pups were fully susceptible to CPV infection. This may be due to poor adherence of the vaccination schedule to be followed in dams by the canine breeders/owners in India in the absence of any strict regulations/guidelines. According to researchers such as Carmichael [5], Pratelli et al. [16], and Tagushi et al. [17], a $\mathrm{HI}$ titer of $\geq 1: 80$ (equivalent to $\geq 1.9 \mathrm{GM}$ $\log _{10}$ HI titer) was enough to protect the pups against virulent challenge, whereas pups having $\mathrm{HI}$ titer of $<1: 40\left(<1.6 \mathrm{GM} \log _{10}\right.$ HI titer) were fully susceptible (Figure-1). The pups having titer between 1:40 and 1:80 (1.6-1.9 GM $\log _{10}$ HI titer) were considered partially protected. In another study, Carmichael [5] observed that the pups with MDA titers $\geq 1: 20$ (equivalent to $>1.3 \mathrm{GM} \log _{10}$ HI titer) may fail to be immunized successfully and remain susceptible to infection.

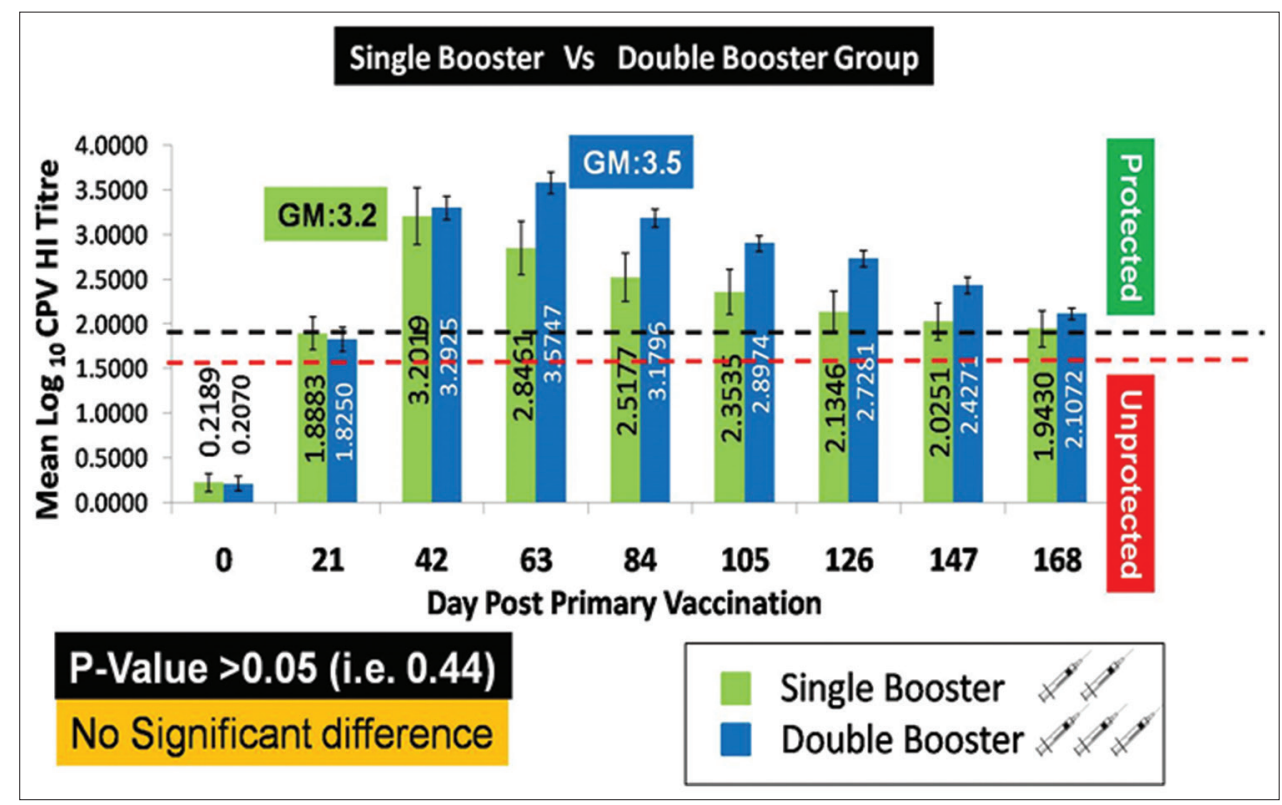

Figure-2: Determination of canine parvovirus antibody titer by hemagglutination inhibition (HI) assay. The value indicates geometric mean $\log 10 \mathrm{HI}$ titer \pm standard error. 


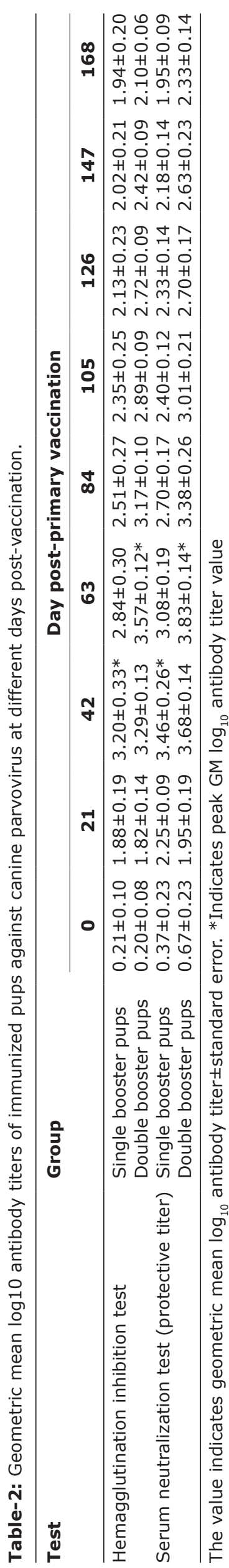

In the present study, the GM $\log _{10}$ HI titers of serum samples at various days post-immunization from the single booster and double booster pups are depicted in Table-2 and Figure-2. The antibody titers of the pups raised substantially almost reaching the protective level $\left(\geq 1: 80 />1.9 \mathrm{GM} \log _{10} \mathrm{HI}\right.$ titer) after primary vaccination in both the single and the double booster vaccinated pups. In the double booster group, the pups showed the peak HI titer (GM: 3.57) at the $63^{\text {rd }}$ day post-primary vaccination, i.e., $21^{\text {st }}$ day after the second booster vaccination followed by a gradual decrease in antibody titer until the end of the study period ( $168^{\text {th }}$ day post-primary vaccination). The pups in the double booster group were also able to maintain the protective HI titers until the end of the study period (GM: 2.10). The pups in the single booster group, on the other hand, reached their peak HI tires (GM: 3.20) at the $42^{\text {nd }}$ day post-primary vaccination, i.e., $21^{\text {st }}$ day after the first booster vaccination, which was slightly lower when compared to the double booster group. The pups in the single booster group were also able to maintain the protective titer at the end of the study period (GM: 1.94) though the titers were found to be slightly lower than the double booster group. Although the difference in HI titers between the two groups of the pups was not statistically significant $(p>0.05$, i.e., 0.44), the pups under the double booster group maintained a better and higher immune response than the single booster group. Therefore, it was inferred from this study that the second booster vaccination will be extremely useful to attain a higher antibody titer for a prolonged period.

\section{SNT}

The $\log _{10}$ GM neutralizing antibody titers, the single and double booster pups at different days post-vaccination, are displayed in Table-2 and Figure-3.

In SNT, the pups under the double booster group showed the peak SN titer (GM: 3.83) at the $63^{\text {rd }}$ day post-primary vaccination, i.e., $21^{\text {st }}$ day after the second booster vaccination followed by a gradual decrease in antibody titer until the end of the study period. In contrast, the pups in the single booster group reached their peak SN titer (GM: 3.46 ) at the $42^{\text {nd }}$ day post-primary vaccination, i.e., $21^{\text {st }}$ day after the first booster vaccination which was slightly lower when compared to the double booster group. Although the difference in SN titers between the two groups of the pups was not statistically significant ( $p>0.05$, i.e., 0.39 ), the pups under the double booster group maintained a better and higher immune response than the pups under the single booster group.

As the SN titers were found comparable with that of the HI titers, there was enough evidence to believe that the antibody titers of the vaccinated pups were protective in nature. Although there was no statistical significance $(p>0.05$, i.e., 0.53$)$ between the test results of HI and SNT, SN titers were comparatively higher due to its higher sensitivity (Figure-4). 


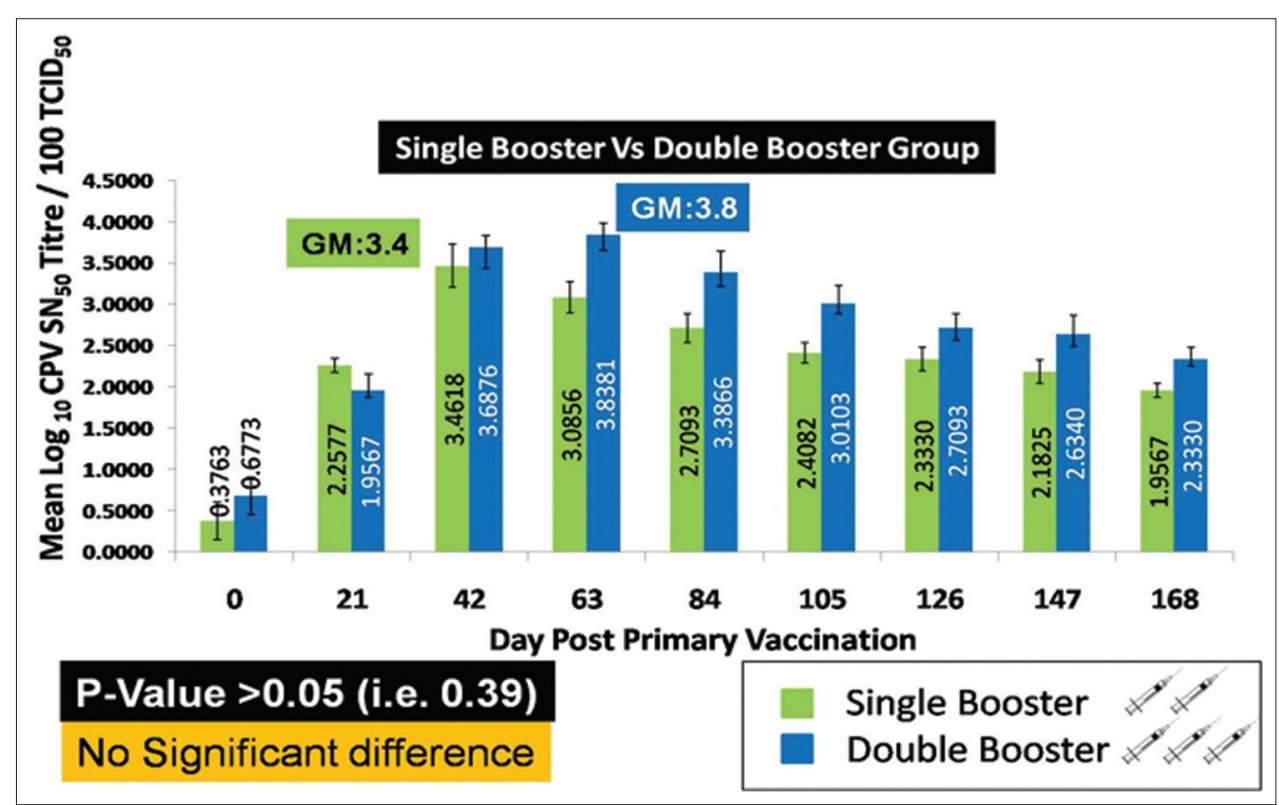

Figure-3: Determination of canine parvovirus-neutralizing antibody titer by serum neutralization test (SNT). The value indicates geometric mean log10 SNT titer \pm standard error.

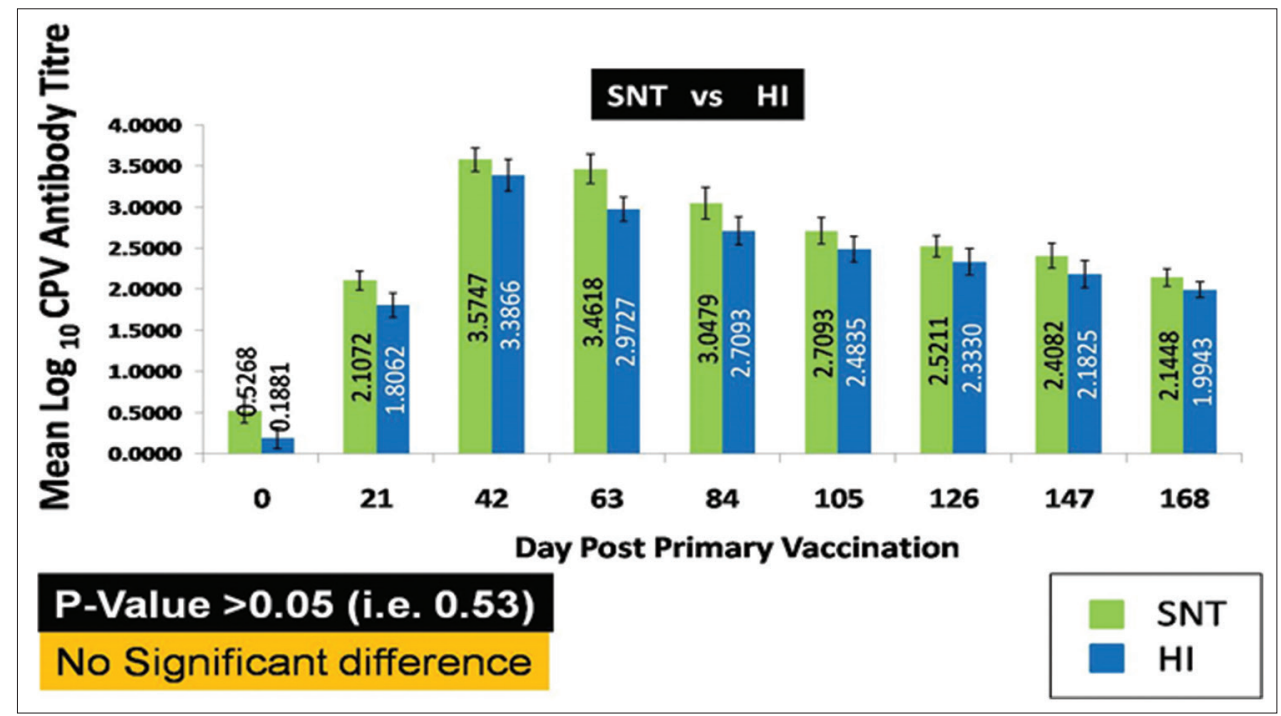

Figure-4: Comparison of the test results of hemagglutination inhibition and serum neutralization tests. The value indicates geometric mean log10 antibody titer \pm standard error.

Therefore, the commercially available CPV-2 based vaccine was found effective in providing protective immunity as indicated by HI and SNT. The results of SNT also indicated that the current vaccine strain $(\mathrm{CPV}-2)$ is capable of neutralizing the prevalent field strain (CPV-2a) in India, as a field CPV-2a strain was used for conducting SNT.

\section{Conclusion}

In this study, serum samples were subjected to $\mathrm{HI}$ and SNT for measuring the CPV antibody titers from 27 healthy pups following primary and booster $\mathrm{CPV}$ vaccinations. The pups were categorized into two groups, the single booster and the double booster pups. The antibody titers in the double booster pups reached their peaks at the $63^{\text {rd }}$ day post-vaccination with GM of 3.57. The antibody titers in the single booster pups reached their peaks at the $42^{\text {nd }}$ day post-vaccination with a lower GM of 3.20. The double booster pups maintained a higher immune response than the single booster pups though the difference in titers was not statistically significant. SNT results indicated that the raised antibody titers were protective in nature though there was no statistical significance between HI assay and SNT. Therefore, the second booster vaccination may be useful in maintaining the protective titer for a prolonged period.

\section{Authors' Contributions}

JV, MVS, and HKM were involved in the design of this research work. JV performed the research. HKM monitored all the activities being a supervisor. MVS, PXA, VP, and JT assisted in this research work. MVS, JV, and HKM drafted and revised the 
manuscript. All authors read and approved the final manuscript.

\section{Acknowledgments}

The authors are thankful to Dean, Rajiv Gandhi Institute of Veterinary Education and Research, Puducherry, India, for providing necessary funds and facilities to carry out this study.

\section{Competing Interests} interests.

The authors declare that they have no competing

\section{Publisher's Note}

Veterinary World remains neutral with regard to jurisdictional claims in published institutional affiliation.

\section{References}

1. Appel, M.J., Scott, F.W. and Carmichael, L.E. (1979) Isolation and immunization studies of a canine parvo-like virus from dogs with hemorrhagic enteritis. Vet. Rec., 105(8): 156-159.

2. Buonavoglia, C., Tollis, M., Buonavoglia, D. and Puccini, A (1992) Response of pups with maternally derived antibody to modified-live canine parvovirus vaccine. Comp. Immunol. Microbiol. Infect. Dis., 15(4): 281-283.

3. Sassa, N., Nalubamba, K.S., Kandawire, E. and Siwila, J. (2016) Seroprevalence of Canine parvovirus in dogs in Lusaka district, Zambia-Hindawi. J. Vet. Med., 2016, Article ID 9781357

4. Riedl, M., Truyen, U., Reese, S. and Hartmann, K. (2015) Prevalence of antibodies to Canine parvovirus and reaction to vaccination in client-owned, healthy dogs. Vet. Record, 177(23): 597

5. Carmichael, L.E. (1999) Canine viral vaccines at a turning point-a personal perspective. Adv. Vet. Med., 41: 289-307.

6. Nova, B.V., Cunha, E., Sepúlveda, N., Oliveira, M., Braz, B.S., Tavares, L., Almeida, V. and Gil, S. (2018) Evaluation of the humoral immune response induced by vaccination for Canine distemper and Parvovirus: A pilot study. BMC Vet. Res., 14(1): 348-355.

7. Decaro, N., Desario, C., Campolo, M., Elia, G., Martella, V., Ricci, D., Lorusso, E. and Buonavoglia, C. (2005) Clinical and virological findings in pups naturally infected by
Canine parvovirus Type 2 Glu-426 mutant. J. Vet. Diagn Invest., 17(2): 133-138.

8. Decaro, N., Elia, G., Desario, C., Roperto, S., Martella, V., Campolo, M., Lorusso, A., Cavalli, A. and Buonavoglia, C. (2006) A minor groove binder probe real-time PCR assay for discrimination between Type 2-based vaccines and field strains of Canine parvovirus. J. Virol. Methods, 136(1-2): 65-70.

9. Ellis, J., Gow, S., Rhodes, C., Lacoste, S., Kong, L., Musil, K. and Snead, E. (2016) Serum antibody responses to vaccinal antigens in lean and obese geriatric dogs. Can . Vet. J., 57(5): 531-534.

10. Kelly, W.R. (1978) An enteric disease of dogs resembling feline panleukopenia. Aust. Vet. J., 54(12): 593.

11. Martella, V., Cavalli, A., Decaro, N., Elia, G., Desario, C., Campolo, M., Bozzo, G., Tarsitano, E. and Buonavoglia, C. (2005) Immunogenicity of an intranasally administered modified live canine parvovirus Type $2 \mathrm{~b}$ vaccine in pups with maternally derived antibodies. Clin. Diagn. Lab. Immunol., 12(10): 1243-1245.

12. Mittal, M., Chakravarti, S., Mohapatra, J.K., Chung, P.K., Dubey, R., Narwal, P.S., Kumar, A., Churamani, C.P. and Kanwar, N.S. (2014) Molecular typing of canine parvovirus strains circulating from 2008-2012 in an organized kennel in India reveals the possibility of vaccination failure. Infect. Genet. Evol., 23: 1-6.

13. Nookala, M., Mukhopadhyay, H.K., Amsaveni, S., Antony, P.X., Thanislass, J., Vivek, S.V.M. and Pillai, R.M. (2016) Full-length VP2 gene analysis of Canine parvovirus reveals the emergence of newer variants in India. Acta. Microbiol. Immunol. Hung., 63(4): 411-426.

14. Mila, H., Grellet, A., Desario, C. Feugier, A., Decaro, N., Buonavoglia, C. and Maillard. S.C. (2014) Protection against canine parvovirus Type 2 infection in puppies by colostrum-derived antibodies. J. Nutr. Sci., 3(54): 1-4.

15. Wilson, S. and Salt, J. (2014) Vaccination of dogs with Canine parvovirus Type $2 \mathrm{~b}(\mathrm{CPV}-2 \mathrm{~b})$ induces neutralizing antibody responses to CPV-2a and CPV-2c. Vaccine, 32(42): 5420-5424.

16. Pratelli, A., Cavalli, A., Martella, V., Tempesta, M., Decaro, N., Carmichael, L.E. and Buonavoglia, C. (2001) Canine parvovirus (CPV) vaccination: Comparison of neutralizing antibody responses in pups after inoculation with CPV2 or CPV2b modified live virus vaccine. Clin. Diag. Lab. Immunol., 8(3): 612-615.

17. Tagushi, M., Namikawa, K., Maeuo, T., Orito, K., Lynch, J. and Sahara, H. (2012) Booster effect of canine distemper, Canine parvovirus infection and infectious canine hepatitis combination vaccine in domesticated adult dogs. Microbiol. Immunol., 56(8): 579-582. 